\title{
Total synthesis of sannanine and analogues thereof
}

\author{
BADHER NAVEEN and RAJAGOPAL NAGARAJAN* (D) \\ School of Chemistry, University of Hyderabad, Hyderabad, Telangana 500 046, India \\ E-mail: nagaindole@gmail.com
}

MS received 15 January 2018; revised 14 February 2018; accepted 14 February 2018; published online 2 March 2018

\begin{abstract}
The first total synthesis of Sannanine has been accomplished with an overall $30 \%$ yield in a concise manner. The key strategies involve Friedländer quinoline synthesis, demethylation, in situ oxidation and amination process.
\end{abstract}

Keywords. Total synthesis; natural products; Friedländer synthesis; sannanine.

\section{Introduction}

Quinoline-5,8-dione is one of the most important cores, found in many natural products as well as in biologically potent molecules ${ }^{1}$ (Figure 1). The potential of quinoline-5,8-dione has been proved in a variety of fields. In general, quinoline-5,8-dione shows the predominant biological activities such as anticancer, antimalarial, anti-inflammatory, and antifungal. ${ }^{1 \mathrm{a}, 2} \mathrm{In}$ addition, quinolone-5,8-dione has very good tenancy to form complexes with transition metals which result in dramatic changes in biological as well as photo-physical properties of quinoline-5,8-dione. Due to bathochromic shift of the metal complex of the quinolone-5,8-dione, quinoline-5,8-dione core has also revealed its potential in 'colour formers'. ${ }^{3}$ Subsequently quinoline-5,8-dione is also used as a fundamental core in the synthesis of many natural products as well as biological efficient molecules. In short, quinoline-5,8-dione has a vital role in most of the fields such as synthetic, biological, and material chemistry. ${ }^{4}$

Sannanine (1) (Figure 1), an anticancer agent, was isolated from Streptomyces sannanensis in 2009 by Han and co-workers and it was found that sannanine exhibits anticancer activity against the four cancer cell lines, such as BGC823, PANC1, HepG2 and $\mathrm{H} 460$ with $\mathrm{IC}_{50}$ values of $6.6,5.8,3.1$ and $1.8 \mu \mathrm{M}$, respectively. ${ }^{5}$ Due to excellent biological activity of sannanine, there is an urgency for its synthesis. Till date, there is no synthetic report on sannanine natural product. Due to more efficacy of the quinoline-5,8-dione core, the natural product

\footnotetext{
*For correspondence
}

sannanine was chosen as an our target molecule, as a part of ongoing natural product synthesis.

\section{Experimental}

\subsection{General}

All the chemicals were used as purchased (Sigma Aldrich \& Avra Synthesis P. Ltd., India) for the reaction without any purification. As per standard procedures, all solvents were dried for the reactions. In addition, highly moisture sensitive reactions were performed under nitrogen atmosphere. All the newly synthesized compounds were characterized by NMR in which $\mathrm{CDCl}_{3}$ and DMSO- $d_{6}$ were used as solvents. In ${ }^{1} \mathrm{H}$ NMR, $7.26 \mathrm{ppm}$ and $2.53 \mathrm{ppm}$ peaks were fixed for $\mathrm{CDCl}_{3}$ and DMSO- $d_{6}$ solvents, respectively. All the compounds were further characterized HRMS and IR spectral analysis. For all the newly synthesized by solid compounds, melting points were determined.

\subsection{Synthetic procedure for}

5,8-dimethoxy-2,3-dimethylquinoline (3) and

2-ethyl-5,8-dimethoxyquinoline (6)

2-Amino-3,6-dimethoxybenzaldehyde (5 g, $0.0275 \mathrm{~mol}$ ) was taken in EtOH $(10 \mathrm{~mL})$. Then, $10 \%$ ethanolic $\mathrm{KOH}(1.1$ equiv.) was added to the reaction mixture and stirred for 12 $\mathrm{h}$ at $90^{\circ} \mathrm{C}$ under $\mathrm{N}_{2}$ atmosphere. The completion of reaction was monitored by thin layer chromatography (TLC). After completion of reaction, the reaction mixture was poured into water $(100 \mathrm{~mL})$ and extracted with EtOAc $(4 \times 50 \mathrm{~mL})$. The crude product was purified by column chromatography using hexane/EtOAc solvent mixture. Finally, 5,8-dimethoxy-2,3dimethylquinoline was obtained as a colourless solid in $62 \%$ yield. $\mathrm{R}_{\mathrm{f}}=0.23(20 \%$ EtOAc/Hexane $)$; M.p.: $126^{\circ} \mathrm{C}$; IR 


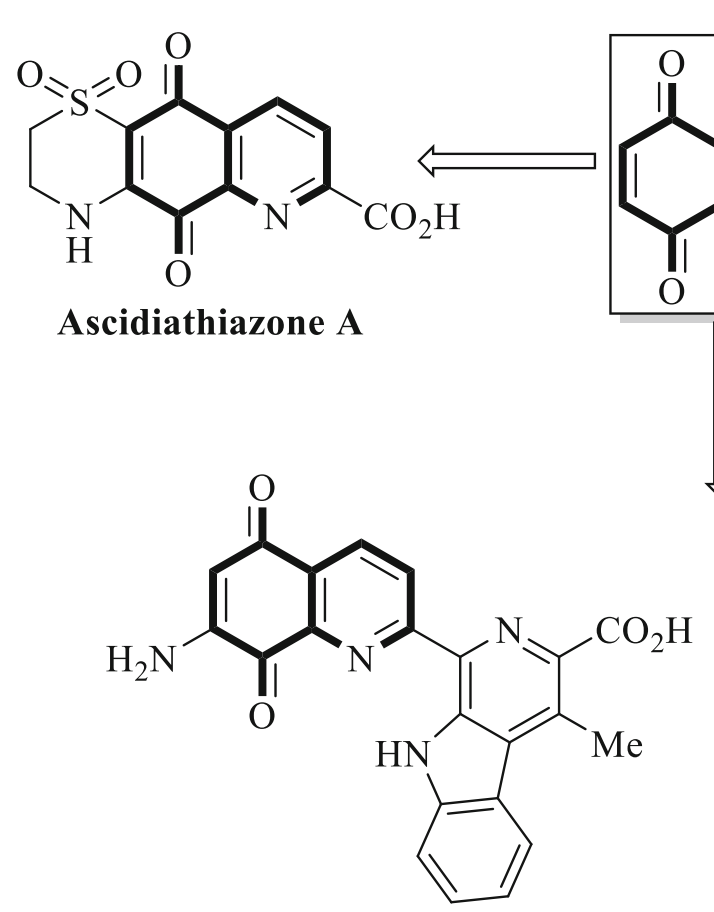

Lavendamycin<smiles>COC1=C(N)C(=O)c2nc(-c3nc(C(=O)O)c(C)c(-c4ccc(OC)c(OC)c4O)c3C)ccc2C1=O</smiles>

Figure 1. Structure of quinoline-5,8-dione, sannanine $\mathbf{1}$ and related natural products.

(KBr): $v_{\max } 3586,3484,3004,2941,2915,2815,2835,1621$, $1609,1482,1467,1454,1426,1404 \mathrm{~cm}^{-1} ;{ }^{1} \mathrm{H} \mathrm{NMR}\left(\mathrm{CDCl}_{3}\right.$, $400 \mathrm{MHz}): \delta 8.16(\mathrm{~s}, 1 \mathrm{H}), 6.77(\mathrm{~d}, J=8.4 \mathrm{~Hz}, 1 \mathrm{H}), 6.60(\mathrm{~d}$, $J=8.4 \mathrm{~Hz}, 1 \mathrm{H}), 3.96(\mathrm{~s}, 3 \mathrm{H}), 3.86(\mathrm{~s}, 3 \mathrm{H}), 2.68$ (s, 3H), 2.38 $(\mathrm{s}, 3 \mathrm{H}) ;{ }^{13} \mathrm{C} \mathrm{NMR}\left(\mathrm{CDCl}_{3}, 100 \mathrm{MHz}\right): \delta 158.3,148.9,148.2$, 138.6, 130.1, 129.8, 120.6, 105.6, 102.8, 55.9, 55.6, 23.8, 19.6; HRMS (ESI): $\mathrm{m} / \mathrm{z}$ calcd. for $\mathrm{C}_{13} \mathrm{H}_{15} \mathrm{NO}_{2}[\mathrm{M}+\mathrm{H}]^{+}$: 218.1182, found: 218.1185 .

2.2a 2-Ethyl-5,8-dimethoxyquinoline(6): Yield:34\%; brown coloured liquid; $\mathrm{R}_{\mathrm{f}}=0.28$ (20\% EtOAc/Hexane); IR (KBr): $v_{\max } 3553,3466,3010,2923,2910,2715,2635,1631$, 1619, 1475, 1467, 1434, 1416, $\mathrm{cm}^{-1} ;{ }^{1} \mathrm{H} \mathrm{NMR}\left(\mathrm{CDCl}_{3}, 400\right.$ $\mathrm{MHz}): \delta 8.47(\mathrm{~d}, J=8.6 \mathrm{~Hz}, 1 \mathrm{H}), 7.36(\mathrm{~d}, J=8.6 \mathrm{~Hz}$, $1 \mathrm{H}), 6.91(\mathrm{~d}, J=8.5 \mathrm{~Hz}, 1 \mathrm{H}), 6.70(\mathrm{~d}, J=8.5 \mathrm{~Hz}$, $1 \mathrm{H}), 4.03$ (s, 3H), 3.94 (s, 3H), 3.08 (q, $J=7.5 \mathrm{~Hz}, 2 \mathrm{H})$, $1.40(\mathrm{t}, J=7.3 \mathrm{~Hz}, 3 \mathrm{H}) ;{ }^{13} \mathrm{C} \mathrm{NMR}\left(\mathrm{CDCl}_{3}, 100 \mathrm{MHz}\right)$ : $\delta 163.4,148.9,148.6,139.9,131.0,120.1,119.9,106.8$, 102.7, 55.9, 55.5, 32.3, 14.1; HRMS (ESI): m/z calcd. for $\mathrm{C}_{13} \mathrm{H}_{15} \mathrm{NO}_{2}[\mathrm{M}+\mathrm{H}]^{+}:$218.1182, found: 218.1174 .

\subsection{Synthetic procedure for}

\section{2,3-dimethylquinoline-5,8-dione (2)}

5,8-Dimethoxy-2,3-dimethylquinoline (2 g, $0.0092 \mathrm{~mol}$ ) was taken in $\mathrm{CH}_{3} \mathrm{CN}(15 \mathrm{~mL})$. This was followed by addition of aq. ceric ammonium nitrate $(\mathrm{CAN})(0.0229 \mathrm{~mol})$ to the reaction mixture at room temperature and allowed to stir for $1 \mathrm{~h}$. The reaction was monitored by TLC. After completion of reaction, the reaction mixture was poured in water
$(50 \mathrm{~mL})$ and extracted with EtOAc $(5 \times 20 \mathrm{~mL})$. The crude product was further used for the next reaction without purification. Yield: $83 \%$ as a red coloured solid; $\mathrm{R}_{\mathrm{f}}=0.23(50 \%$ EtOAc/Hexane); M.p.: $102^{\circ} \mathrm{C}$; IR (KBr): $v_{\max } 1655,1640$, 1461, 1423, 1400, 1390, $1350 \mathrm{~cm}^{-1}$; ${ }^{1} \mathrm{H} \mathrm{NMR}\left(\mathrm{CDCl}_{3}, 400\right.$ $\mathrm{MHz}): \delta 8.08(\mathrm{~s}, 1 \mathrm{H}), 7.07(\mathrm{~d}, J=10.4 \mathrm{~Hz}, 1 \mathrm{H}), 6.98(\mathrm{~d}$, $J=10.3 \mathrm{~Hz}, 1 \mathrm{H}), 2.71(\mathrm{~s}, 3 \mathrm{H}), 2.46(\mathrm{~s}, 3 \mathrm{H}) ;{ }^{13} \mathrm{C} \mathrm{NMR}$ $\left(\mathrm{CDCl}_{3}, 100 \mathrm{MHz}\right): \delta 185.1,183.6,164.2,144.8,138.8,137.6$, 137.6, 134.4, 127.4, 23.4, 19.7; HRMS (ESI): m/z calcd. for $\mathrm{C}_{11} \mathrm{H}_{9} \mathrm{NO}_{2}[\mathrm{M}+\mathrm{H}]^{+}: 188.0712$, found: 188.0717 .

\subsection{General synthetic procedure for the compounds 1 and $8-19$}

2,3-Dimethylquinoline-5,8-dione (0.0004 mol) was taken in EtOH ( $2 \mathrm{~mL})$. Subsequently, the amines (1.5 equiv.) were added to the reaction mixture and allowed to stir for $2 \mathrm{~h}$ at room temperature. After completion of reaction, the solvent was evaporated under reduced pressure and finally the crude products were purified by column chromatography using hexanes/EtOAc mixtures.

\section{4a 2,3-Dimethyl-6-(methylamino) quinolone-5,8-}

dione (1): Yield: $49 \%$ (in two steps) as a red colored solid; $\mathrm{R}_{\mathrm{f}}=0.44$ (EtOAc/Hexane); M.p.: $198^{\circ} \mathrm{C}$; IR $(\mathrm{KBr})$ : $v_{\max } 3288,1683,1627,1596,1550,1505,1453,1414$, 1372, 1333, 1290, 1234, $1200 \mathrm{~cm}^{-1} ;{ }^{1} \mathrm{H} \mathrm{NMR}\left(\mathrm{CDCl}_{3}, 500\right.$ $\mathrm{MHz}): \delta 7.99(\mathrm{~s}, 1 \mathrm{H}), 5.97(\mathrm{~s}, 1 \mathrm{H}), 5.80(\mathrm{~s}, 1 \mathrm{H}), 2.90(\mathrm{~d}$, $J=4.5 \mathrm{~Hz}, 3 \mathrm{H}), 2.66(\mathrm{~s}, 3 \mathrm{H}), 2.39(\mathrm{~s}, 3 \mathrm{H}) ;{ }^{13} \mathrm{C} \mathrm{NMR}$ $\left(\mathrm{CDCl}_{3}, 125 \mathrm{MHz}\right): \delta 181.81,181.80,164.4,148.4,146.8$, 
135.3, 134.3, 125.4, 101.1, 29.1, 23.4, 19.3; HRMS (ESI): $\mathrm{m} / \mathrm{z}$ calcd. for $\mathrm{C}_{12} \mathrm{H}_{12} \mathrm{~N}_{2} \mathrm{O}_{2}[\mathrm{M}+\mathrm{H}]^{+}$: 217.0978, found: 217.0978 .

\section{4b 2,3-Dimethyl-7-(methylamino)quinolone-5,8-}

dione (7): Yield: $27 \%$ (in two steps) as a red colored solid; $\mathrm{R}_{\mathrm{f}}=0.16$ (50\% EtOAc/Hexane); M.p.: $192^{\circ} \mathrm{C}$; IR $(\mathrm{KBr}): v_{\max } 3331,1697,1596,1550,1505,1453,1415,1372$, 1333, 1270, 1234, 1200, $1168 \mathrm{~cm}^{-1} ;{ }^{1} \mathrm{H} \mathrm{NMR}\left(\mathrm{CDCl}_{3}, 400\right.$ $\mathrm{MHz}): \delta 8.09(\mathrm{~s}, 1 \mathrm{H}), 6.14(\mathrm{~s}, 1 \mathrm{H}), 5.69(\mathrm{~s}, 1 \mathrm{H}), 2.95(\mathrm{~d}$, $J=5.4 \mathrm{~Hz}, 3 \mathrm{H}), 2.65(\mathrm{~s}, 3 \mathrm{H}), 2.42(\mathrm{~s}, 3 \mathrm{H}) ;{ }^{13} \mathrm{C} \mathrm{NMR}$ $\left(\mathrm{CDCl}_{3}, 100 \mathrm{MHz}\right): \delta 182.2,180.2,161.8,149.1,144.0,138.1$, 134.4, 129.1, 99.5, 29.2, 23.0, 19.7; HRMS (ESI): m/z calcd. for $\mathrm{C}_{12} \mathrm{H}_{12} \mathrm{~N}_{2} \mathrm{O}_{2}[\mathrm{M}+\mathrm{Na}]^{+}$: 239.0795, found: 239.0795 .

\section{4c 6-(Benzylamino)-2,3-dimethylquinoline-5,8-}

dione (8): Yield: $51 \%$ (in two steps) as a red colored solid; $\mathrm{R}_{\mathrm{f}}=0.16$ (50\% EtOAc/Hexane); M.p.: $180^{\circ} \mathrm{C}$; IR (KBr): $v_{\max } 3289,1673,1624,1599,1585,1552$, 1498, 1445, 1381, 1332, 1280, 1228, $1211 \mathrm{~cm}^{-1} ;{ }^{1} \mathrm{H} \mathrm{NMR}\left(\mathrm{CDCl}_{3}, 400 \mathrm{MHz}\right)$ : $\delta 8.02(\mathrm{~s}, 1 \mathrm{H}), 7.34(\mathrm{~m}, 5 \mathrm{H}), 6.18(\mathrm{~s}, 1 \mathrm{H}), 5.89(\mathrm{~s}, 1 \mathrm{H}), 4.37$ $(\mathrm{d}, J=5.5 \mathrm{~Hz}, 2 \mathrm{H}), 2.69(\mathrm{~s}, 3 \mathrm{H}), 2.41(\mathrm{~s}, 3 \mathrm{H}) ;{ }^{13} \mathrm{C} \mathrm{NMR}$ $\left(\mathrm{CDCl}_{3}, 100 \mathrm{MHz}\right): \delta 181.9,181.8,164.5,147.1,146.6,135.6$, 135.4, 134.3, 129.0, 128.2, 127.7, 125.5, 102.2, 46.8, 23.5, 19.4; HRMS (ESI): $\mathrm{m} / \mathrm{z}$ calcd. for $\mathrm{C}_{18} \mathrm{H}_{16} \mathrm{~N}_{2} \mathrm{O}_{2}[\mathrm{M}+\mathrm{H}]^{+}$: 293.1291, found: 293.1288.

\section{4d 7-(Benzylamino)-2,3-dimethylquinoline-5,8-}

dione (9): Yield: $24 \%$ (in two steps) as a red colored solid; $\mathrm{R}_{\mathrm{f}}=0.40$ (50\% EtOAc/Hexane); M.p.: $168^{\circ} \mathrm{C}$; IR (KBr): $v_{\max } 3245,1694,1598,1550,1454,1440,1415,1378,1353$, $1335,1273,1233,1202,1192 \mathrm{~cm}^{-1} ;{ }^{1} \mathrm{H} \mathrm{NMR}\left(\mathrm{CDCl}_{3}, 400\right.$ $\mathrm{MHz}): \delta 8.10(\mathrm{~s}, 1 \mathrm{H}), 7.32(\mathrm{~m}, 5 \mathrm{H}), 6.38(\mathrm{~s}, 1 \mathrm{H}), 5.77(\mathrm{~s}$, $1 \mathrm{H}), 4.41(\mathrm{~d}, J=5.8 \mathrm{~Hz}, 2 \mathrm{H}), 2.68(\mathrm{~s}, 3 \mathrm{H}), 2.44(\mathrm{~s}, 3 \mathrm{H}) ;{ }^{13} \mathrm{C}$ $\mathrm{NMR}\left(\mathrm{CDCl}_{3}, 100 \mathrm{MHz}\right): \delta 182.4,180.3,162.0,147.9,144.0$, 138.2, 135.6, 134.4, 129.0, 128.2, 127.6, 100.7, 46.9, 23.0, 19.8; HRMS (ESI): $\mathrm{m} / \mathrm{z}$ calcd. for $\mathrm{C}_{18} \mathrm{H}_{16} \mathrm{~N}_{2} \mathrm{O}_{2}[\mathrm{M}+\mathrm{Na}]^{+}$: 315.1109, found: 315.1107 .

\section{4e 2,3-Dimethyl-6-(phenethylamino)quinolone-}

5,8-dione (10): Yield: $66 \%$ (in two steps) as a red colored solid; $\mathrm{R}_{\mathrm{f}}=0.49$ (EtOAc); M.p.: $206^{\circ} \mathrm{C}$; IR $(\mathrm{KBr}): v_{\max }$ $3345,1688,1614,1559,1538,1460,1375,1342,1292,1235$, 1200, 1156, $1120 \mathrm{~cm}^{-1} ;{ }^{1} \mathrm{H} \mathrm{NMR}\left(\mathrm{CDCl}_{3}, 400 \mathrm{MHz}\right): \delta 7.99$ $(\mathrm{s}, 1 \mathrm{H}), 7.33(\mathrm{t}, J=7.4 \mathrm{~Hz}, 2 \mathrm{H}), 7.27(\mathrm{~d}, J=7.8 \mathrm{~Hz}, 1 \mathrm{H})$, $7.22(\mathrm{~d}, J=7.2 \mathrm{~Hz}, 2 \mathrm{H}), 5.92(\mathrm{~s}, 1 \mathrm{H}), 5.89$ (s, 1H), 3.46 $(\mathrm{q}, J=6.9 \mathrm{~Hz}, 2 \mathrm{H}), 2.98(\mathrm{t}, J=6.9 \mathrm{~Hz}, 2 \mathrm{H}), 2.69(\mathrm{~s}, 3 \mathrm{H})$, $2.40(\mathrm{~s}, 3 \mathrm{H}) ;{ }^{13} \mathrm{C} \mathrm{NMR}\left(\mathrm{CDCl}_{3}, 100 \mathrm{MHz}\right): \delta 181.89,181.80$, 164.4, 147.1, 146.7, 137.6, 135.3, 134.3, 128.8, 128.6, 126.9, 125.4, 101.5, 43.6, 34.2, 23.5, 19.3; HRMS (ESI): m/z calcd. for $\mathrm{C}_{19} \mathrm{H}_{18} \mathrm{~N}_{2} \mathrm{O}_{2}[\mathrm{M}+\mathrm{H}]^{+}:$307.1447, found: 307.1448 .

2.4f 2,3-Dimethyl-7-(phenethylamino)quinolone-5,8dione (11): Yield: $30 \%$ (in two steps) as a red colored solid; $\mathrm{R}_{\mathrm{f}}=0.56$ (EtOAc); M.p.: $148^{\circ} \mathrm{C}$; IR (KBr): $v_{\max }$ 3331, 1698, 1604, 1552, 1508, 1449, 1370, 1332, 1272, 1235, $1202,1146,1106 \mathrm{~cm}^{-1} ;{ }^{1} \mathrm{H} \mathrm{NMR}\left(\mathrm{CDCl}_{3}, 400 \mathrm{MHz}\right): \delta 8.11$ $(\mathrm{s}, 1 \mathrm{H}), 7.34(\mathrm{t}, J=7.2 \mathrm{~Hz}, 2 \mathrm{H}), 7.28(\mathrm{~d}, J=3.8 \mathrm{~Hz}, 1 \mathrm{H})$, $7.23(\mathrm{~d}, J=7.1 \mathrm{~Hz}, 2 \mathrm{H}), 6.12(\mathrm{~s}, 1 \mathrm{H}), 5.77(\mathrm{~s}, 1 \mathrm{H}), 3.49$ $(\mathrm{q}, J=6.7 \mathrm{~Hz}, 2 \mathrm{H}), 3.00(\mathrm{t}, J=7.4 \mathrm{~Hz}, 2 \mathrm{H}), 2.67(\mathrm{~s}, 3 \mathrm{H})$, $2.44(\mathrm{~s}, 3 \mathrm{H}) ;{ }^{13} \mathrm{C} \mathrm{NMR}\left(\mathrm{CDCl}_{3}, 100 \mathrm{MHz}\right): \delta 182.3,180.2$, 161.9, 147.9, 144.0, 138.1, 137.7, 134.4, 129.0, 128.8, 128.6, 126.9, 99.9, 43.9, 34.3, 23.0, 19.7; HRMS (ESI): m/z calcd. for $\mathrm{C}_{19} \mathrm{H}_{18} \mathrm{~N}_{2} \mathrm{O}_{2}[\mathrm{M}+\mathrm{Na}]^{+}$: 329.1265, found: 329.1265 .

\section{$2.4 \mathrm{~g}$ 6-((4-Methoxyphenethyl)amino)-2,3-}

dimethylquinoline-5,8-dione (12): Yield: $47 \%$ (in two steps) as a red colored solid; $\mathrm{R}_{\mathrm{f}}=0.14$ (50\% EtOAc/Hexane); M.p.: $214{ }^{\circ} \mathrm{C}$; IR (KBr): $v_{\max } 3376,1687,1605,1553,1504$, $1467,1435,1377,1342,1312,1282,1240,1197,1183 \mathrm{~cm}^{-1}$; ${ }^{1} \mathrm{H} \mathrm{NMR}\left(\mathrm{CDCl}_{3}, 400 \mathrm{MHz}\right): \delta 7.98(\mathrm{~s}, 1 \mathrm{H}), 7.11(\mathrm{~d}, J=$ $7.5 \mathrm{~Hz}, 2 \mathrm{H}$ ), 6.85 (d, $J=7.4 \mathrm{~Hz}, 2 \mathrm{H}), 5.87$ (s, 2H) (Including both $-\mathrm{NH}$ proton and $-\mathrm{CH}\left(7^{\text {th }}\right.$ position) proton $), 3.77(\mathrm{~s}$, $3 \mathrm{H}), 3.40(\mathrm{q}, J=5.8 \mathrm{~Hz}, 2 \mathrm{H}), 2.90(\mathrm{t}, J=6.3 \mathrm{~Hz}, 2 \mathrm{H})$, $2.67(\mathrm{~s}, 3 \mathrm{H}), 2.39$ (s, 3H); ${ }^{13} \mathrm{C} \mathrm{NMR}\left(\mathrm{CDCl}_{3}, 100 \mathrm{MHz}\right)$ : $\delta$ 181.9, 181.8, 164.4, 158.5, 147.1, 146.7, 135.3, 134.3, $134.2,129.6,125.4,114.3,101.4,55.3,43.8,33.4,23.5$, 19.4; HRMS (ESI): m/z calcd. for $\mathrm{C}_{20} \mathrm{H}_{20} \mathrm{~N}_{2} \mathrm{O}_{3}[\mathrm{M}+\mathrm{H}]^{+}$: 337.1553, found: 337.1556 .

\section{4h 7-((4-Methoxyphenethyl)amino)-2,3-}

dimethylquinoline-5,8-dione (13): Yield: $22 \%$ (in two steps) as a red colored solid; $\mathrm{R}_{\mathrm{f}}=0.21$ (50\% EtOAc/Hexane); M.p.: $198^{\circ} \mathrm{C}$; IR (KBr): $v_{\max } 3366,1677,1615,1573$, $1500,1477,1425,1370,1345,1315,1282,1251,1187$, $1180 \mathrm{~cm}^{-1} ;{ }^{1} \mathrm{H} \mathrm{NMR}\left(\mathrm{CDCl}_{3}, 400 \mathrm{MHz}\right): \delta 8.12(\mathrm{~s}, 1 \mathrm{H})$, $7.14(\mathrm{~d}, J=8.4 \mathrm{~Hz}, 2 \mathrm{H}), 6.87(\mathrm{~d}, J=8.6 \mathrm{~Hz}, 2 \mathrm{H})$, 6.11(s, 1H), $5.76(\mathrm{~s}, 1 \mathrm{H}), 3.81(\mathrm{~s}, 3 \mathrm{H}), 3.44(\mathrm{q}, J=6.7 \mathrm{~Hz}$, $2 \mathrm{H}), 2.94(\mathrm{t}, J=7.3 \mathrm{~Hz}, 2 \mathrm{H}), 2.67(\mathrm{~s}, 3 \mathrm{H}), 2.44(\mathrm{~s}, 3 \mathrm{H})$; ${ }^{13} \mathrm{C} \mathrm{NMR}\left(\mathrm{CDCl}_{3}, 100 \mathrm{MHz}\right): \delta 182.3,180.2,161.9,158.5$, 147.9, 144.0, 138.1, 134.4, 129.6, 129.6, 129.1, 114.3, 99.9, 55.2, 44.1, 33.4, 23.0, 19.7; HRMS (ESI): m/z calcd. for $\mathrm{C}_{20} \mathrm{H}_{20} \mathrm{~N}_{2} \mathrm{O}_{3}[\mathrm{M}+\mathrm{H}]^{+}:$337.1553, found: 337.1554 .

\section{$2.4 \mathrm{i} \quad 6-((2-(1 H-I n d o l-3-y l)$ ethyl $)$ amino $)-2,3-$}

dimethylquinoline-5,8-dione (14): Yield: $48 \%$ (in two steps) as a red colored solid; $\mathrm{R}_{\mathrm{f}}=0.25$ (50\% EtOAc/Hexane); M.p.: $144^{\circ} \mathrm{C}$; IR (KBr): $v_{\max } 3341,3273,1685,1633,1609$, 1588, 1557, 1504, 1462, 1443, 1370, 1329, 1229, 1271, $1233 \mathrm{~cm}^{-1} ;{ }^{1} \mathrm{H} \mathrm{NMR}\left(\mathrm{CDCl}_{3}, 400 \mathrm{MHz}\right): \delta 8.17(\mathrm{~s}, 1 \mathrm{H})$, $8.12(\mathrm{~s}, 1 \mathrm{H}), 7.63(\mathrm{~d}, J=7.9 \mathrm{~Hz}, 1 \mathrm{H}), 7.41(\mathrm{~d}, J=8.1 \mathrm{~Hz}$, $1 \mathrm{H}), 7.24(\mathrm{t}, J=7.7 \mathrm{~Hz}, 1 \mathrm{H}), 7.17(\mathrm{t}, J=7.5 \mathrm{~Hz}, 1 \mathrm{H}), 7.10$ $(\mathrm{s}, 1 \mathrm{H}), 6.18(\mathrm{~s}, 1 \mathrm{H}), 5.78(\mathrm{~s}, 1 \mathrm{H}), 3.55(\mathrm{q}, J=6.0 \mathrm{~Hz}, 1 \mathrm{H})$, $3.18(\mathrm{t}, J=6.9 \mathrm{~Hz}, 1 \mathrm{H}), 2.67(\mathrm{~s}, 3 \mathrm{H}), 2.44(\mathrm{~s}, 3 \mathrm{H}) ;{ }^{13} \mathrm{C} \mathrm{NMR}$ $\left(\mathrm{CDCl}_{3}, 100 \mathrm{MHz}\right): \delta 182.2,180.8,163.8,148.1,146.5,136.7$, 135.5, 134.1, 127.5, 125.9, 123.5, 121.4, 118.7, 118.6, 111.8, 111.6, 100.5, 43.1, 23.7, 23.4, 19.0; HRMS (ESI): m/z calcd. for $\mathrm{C}_{21} \mathrm{H}_{19} \mathrm{~N}_{3} \mathrm{O}_{2}[\mathrm{M}+\mathrm{H}]^{+}$: 346.1556, found: 346.1557 .

\section{4j 7-((2-(1H-Indol-3-yl)ethyl)amino $)-2,3-$}

dimethylquinoline-5,8-dione (15): Yield: $27 \%$ (in two steps) as a red colored solid; $\mathrm{R}_{\mathrm{f}}=0.32$ (50\% EtOAc/Hexane); M.p.: $150^{\circ} \mathrm{C}$; IR (KBr): $v_{\max } 3229,2927,1682,1630,1601$, 1584, 1557, 1511, 1460, 1443, 1381, 1337, 1301, 1272, 
1244, 1226, $1175 \mathrm{~cm}^{-1} ;{ }^{1} \mathrm{H} \mathrm{NMR}\left(\mathrm{CDCl}_{3}, 400 \mathrm{MHz}\right): \delta$ $8.43(\mathrm{~s}, 1 \mathrm{H}), 8.12(\mathrm{~s}, 1 \mathrm{H}), 7.62(\mathrm{~d}, J=7.7 \mathrm{~Hz}, 1 \mathrm{H}), 7.39$ $(\mathrm{d}, J=7.9 \mathrm{~Hz}, 1 \mathrm{H}), 7.23(\mathrm{t}, J=6.8 \mathrm{~Hz}, 1 \mathrm{H}), 7.15(\mathrm{~d}$, $J=7.4 \mathrm{~Hz}, 1 \mathrm{H}), 7.10(\mathrm{~s}, 1 \mathrm{H}), 6.19(\mathrm{~s}, 1 \mathrm{H}), 5.78(\mathrm{~s}, 1 \mathrm{H}), 3.53$ $(\mathrm{d}, J=6.2 \mathrm{~Hz}, 2 \mathrm{H}), 3.17(\mathrm{t}, J=6.2 \mathrm{~Hz}, 2 \mathrm{H}), 2.66(\mathrm{~s}, 3 \mathrm{H})$, $2.44(\mathrm{~s}, 3 \mathrm{H}) ;{ }^{13} \mathrm{C} \mathrm{NMR}\left(\mathrm{CDCl}_{3}, 100 \mathrm{MHz}\right): \delta 182.3,180.2$, $161.8,148.0,144.0,138.1,136.4,134.4,129.1,126.9,122.3$ (2 Ar-C), 119.6, 118.4, 111.8, 111.4, 99.8, 42.7, 24.1, 22.9, 19.7; HRMS (ESI): $\mathrm{m} / \mathrm{z}$ calcd. for $\mathrm{C}_{21} \mathrm{H}_{19} \mathrm{~N}_{3} \mathrm{O}_{2}[\mathrm{M}+\mathrm{H}]^{+}$: 346.1556, found: 346.1552 .

\section{4k 6-(Allylamino)-2,3-dimethylquinoline-5,8-}

dione (16): Yield: $59 \%$ (in two steps) as a red colored viscous liquid; $\mathrm{R}_{\mathrm{f}}=0.52$ (50\% EtOAc/Hexane); $\mathrm{IR}(\mathrm{KBr})$ : $v_{\max } 3281,1673,1637,1566,1550,1495,1451,1434,1382$, 1334, 1293, 1236, 1203, 1172, $1102 \mathrm{~cm}^{-1} ;{ }^{1} \mathrm{H} \mathrm{NMR}\left(\mathrm{CDCl}_{3}\right.$, $400 \mathrm{MHz}): \delta 7.99(\mathrm{~s}, 1 \mathrm{H}), 5.97(\mathrm{~s}, 1 \mathrm{H}), 5.82(\mathrm{~s}, 1 \mathrm{H}), 5.30(\mathrm{~m}$, $2 \mathrm{H}), 3.81(\mathrm{t}, J=5.5 \mathrm{~Hz}, 2 \mathrm{H}), 2.66(\mathrm{~s}, 3 \mathrm{H}), 2.38(\mathrm{~s}, 3 \mathrm{H})$; ${ }^{13} \mathrm{C} \mathrm{NMR}\left(\mathrm{CDCl}_{3}, 100 \mathrm{MHz}\right): \delta 181.8,181.8,164.3,147.1$, 146.6, 135.3, 134.2, 131.4, 125.4, 118.3, 102.0, 44.8, 23.3, 19.2; HRMS (ESI): $\mathrm{m} / \mathrm{z}$ calcd. for $\mathrm{C}_{14} \mathrm{H}_{14} \mathrm{~N}_{2} \mathrm{O}_{2}[\mathrm{M}+\mathrm{Na}]^{+}$: 265.0952, found: 265.0957.

[Note: Due to very low yield, compound $\mathbf{1 7}$ was only confirmed by HRMS (ESI): m/z calcd. for $\mathrm{C}_{14} \mathrm{H}_{14} \mathrm{~N}_{2} \mathrm{O}_{2}$ $[\mathrm{M}+\mathrm{H}]^{+}:$243.1134, found: 243.1134].

\subsection{2,3-Dimethyl-6-(prop-2-yn-1-ylamino)quinoline-} 5,8-dione (18): Yield: 52\% (in two steps) as a red colored solid; $\mathrm{R}_{\mathrm{f}}=0.30$ (50\% EtOAc/Hexane); M.p.: $156^{\circ} \mathrm{C}$; IR (KBr): $v_{\max } 3291,1676,1647,1568,1555,1505,1454,1424$, 1380, 1344, 1292, 1246, 1205, 1177, $1112 \mathrm{~cm}^{-1} ;{ }^{1} \mathrm{H}$ NMR $\left(\mathrm{CDCl}_{3}, 400 \mathrm{MHz}\right): \delta 8.00(\mathrm{~s}, 1 \mathrm{H}), 7.77(\mathrm{~d}, J=5.5 \mathrm{~Hz}$, $1 \mathrm{H}), 5.79(\mathrm{~s}, 1 \mathrm{H}), 4.03(\mathrm{t}, J=3.0 \mathrm{~Hz}, 2 \mathrm{H}), 3.26(\mathrm{~s}, 1 \mathrm{H})$, $2.55(\mathrm{~s}, 3 \mathrm{H}), 2.37(\mathrm{~s}, 3 \mathrm{H}) ;{ }^{13} \mathrm{C} \mathrm{NMR}\left(\mathrm{CDCl}_{3}, 100 \mathrm{MHz}\right): \delta$ 182.0, 181.1, 163.8, 147.6, 146.1, 135.8, 134.1, 125.9, 102.4, 79.3, 75.0, 31.6, 23.4, 19.1; HRMS (ESI): m/z calcd. for $\mathrm{C}_{14} \mathrm{H}_{12} \mathrm{~N}_{2} \mathrm{O}_{2}[\mathrm{M}+\mathrm{Na}]^{+}$: 263.0796, found: 263.0796.

\section{4m 2,3-Dimethyl-7-(prop-2-yn-1-ylamino)}

quinoline-5,8-dione (19): Yield: $32 \%$ (in two steps) as a red coloured solid; $\mathrm{R}_{\mathrm{f}}=0.39(50 \%$ EtOAc/Hexane $)$; M.p.: $142^{\circ} \mathrm{C}$; IR (KBr): $v_{\max } 3261,1666,1650,1575,1562$, 1532, 1467, 1424, 1374, 1354, 1300, 1256, 1235, 1167, 1115, $1098 \mathrm{~cm}^{-1} ;{ }^{1} \mathrm{H} \mathrm{NMR}\left(\mathrm{CDCl}_{3}, 400 \mathrm{MHz}\right): \delta 8.11(\mathrm{~s}, 1 \mathrm{H}), 6.18$ (s, 1H), $5.84(\mathrm{~s}, 1 \mathrm{H}), 4.03(\mathrm{~d}, J=2.8 \mathrm{~Hz}, 2 \mathrm{H}), 2.67(\mathrm{~s}, 3 \mathrm{H})$, $2.44(\mathrm{~s}, 3 \mathrm{H}), 2.37(\mathrm{~s}, 1 \mathrm{H}) ;{ }^{13} \mathrm{C}$ NMR $\left(\mathrm{CDCl}_{3}, 100 \mathrm{MHz}\right): \delta$ 182.5, 179.9, 162.2, 147.2, 143.9, 138.2, 134.4, 128.8, 101.5, 77.9, 73.5, 32.3, 23.0, 19.7; HRMS (ESI): $\mathrm{m} / \mathrm{z}$ calcd. for $\mathrm{C}_{14} \mathrm{H}_{12} \mathrm{~N}_{2} \mathrm{O}_{2}[\mathrm{M}+\mathrm{Na}]^{+}:$263.0796, found: 263.0801 .

\section{Results and Discussion}

The proposed synthetic diagram for sannanine (1) is depicted in Scheme 1. Sannanine could be easily synthesized from quinoline-5,8-dione 2 by Michael addition of methylamine. Similarly, the 5,8-dione 2 could be obtained using demethylation of the quinoline compound 3. At the initial stage, the quinoline compound 3 could be easily obtained by following the standard Friedländer quinoline synthesis.

The synthesis was commenced with the synthesis of starting precursor 4. Using the standard literature procedure, ortho-amino aldehyde compound 4 was synthesized. ${ }^{6}$ Having enough starting material $\mathbf{4}$ in hand, Friedländer quinoline synthesis ${ }^{7}$ was carried out to synthesize 2,3-dimethyl-5,8-dimethoxy quinoline 3. The compound 4 was treated with ethyl methyl ketone in the presence of $10 \%$ ethanolic $\mathrm{KOH}$ solution to afford the quinoline compound 3 in $62 \%$ yield and 2-ethyl quinoline 6 as a minor product in $34 \%$ yield (overall yield is $96 \%$ ). Then, the compound $\mathbf{3}$ was subjected to demethylation and in situ oxidation to arrive at the dimethyl substituted quinoline-5,8-dione 2 which was treated with methylamine to obtain two regio isomers $\mathbf{1}$ and 7 with $46 \%$ and $27 \%$ yields (overall yield is $73 \%$ ) in two steps, respectively. Out of the two regio isomers (1 \& 7), 6-N-methylamine substituted quinoline-5,8-dione is our desired natural product $\mathbf{1}$.

The spectral data of synthesized sannanine resembles with the reported values of $\mathbf{1}$ under fine shimming NMR. In ${ }^{1} \mathrm{H} \mathrm{NMR}$, the most important characteristic peak is that the methyl peak which should appear as a doublet with respect to NH proton (Table 1). In literature, the two carbonyl peaks for the natural product appeared in 181.78 \& $181.75 \mathrm{ppm}$ in ${ }^{13} \mathrm{C}$ NMR. Moreover, the difference between these two carbonyl peaks is $0.03 \mathrm{ppm}$. Similarly, for the synthesized product $\mathbf{1}$, the peaks belonging to carbonyl groups appeared in 181.80 $\& 181.81 \mathrm{ppm}$ and the difference is $0.01 \mathrm{ppm}$ which are closer to reported values of reported ${ }^{13} \mathrm{C}$ NMR. Based on the above data, the synthesized product 1 was confirmed apart from its regio isomer 7 . As a result, the sannanine 1 was successfully synthesized in $30 \%$ overall yield for the first time using easily affordable starting materials in concise manner.

Subsequent to the synthesis of sannanine 1, the analogues of the natural product $\mathbf{1}$ were synthesized (Scheme 3). To make a library of sannanine 1, a variety of amines have been chosen as one of the starting materials. The synthesis of analogues of sannanine is outlined in Scheme 3. The quinoline-5,8-dione 2 was treated with different amines to obtained the two different regio isomers in moderate to good yields.

Among all the derivatives, phenyethylamine substituted analogues were obtained in good yields $(\mathbf{1 0}(66 \%)$ and $11(30 \%))$. However, in the case of allylamine, there is an isomer, which belongs to 6-substituted analogue 16, achieved in $59 \%$ yield, whereas 7-substituted analogue 17 was found only in trace amount (yield 8\%). 
<smiles>CNC1=CC(=O)c2nc(C)c(C)cc2C1=O</smiles>

Sannanine (1)<smiles>Cc1cc2c(nc1C)C(=O)C=CC2=O</smiles>

2

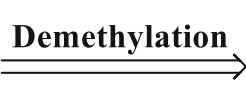<smiles>COc1ccc(OC)c2nc(C)c(C)cc12</smiles>

3

\section{Friedländer quinoline synthesis}<smiles>CCC(C)=O</smiles>

4

Scheme 1. Retrosynthetic analysis of sannanine (1).<smiles>COc1ccc(OC)c(C=O)c1N</smiles>

4
$10 \%$ ethanolic

KOH (1 equiv.)

$90{ }^{0} \mathrm{C}, 12 \mathrm{~h}, \mathrm{~N}_{2}$

5

33 wt. $\%$ aq. $\mathrm{MeNH}_{2}$

3, aq. CAN (2.4 equiv.)

$\mathrm{CH}_{3} \mathrm{CN}, \mathrm{rt}, 1 \mathrm{~h}$

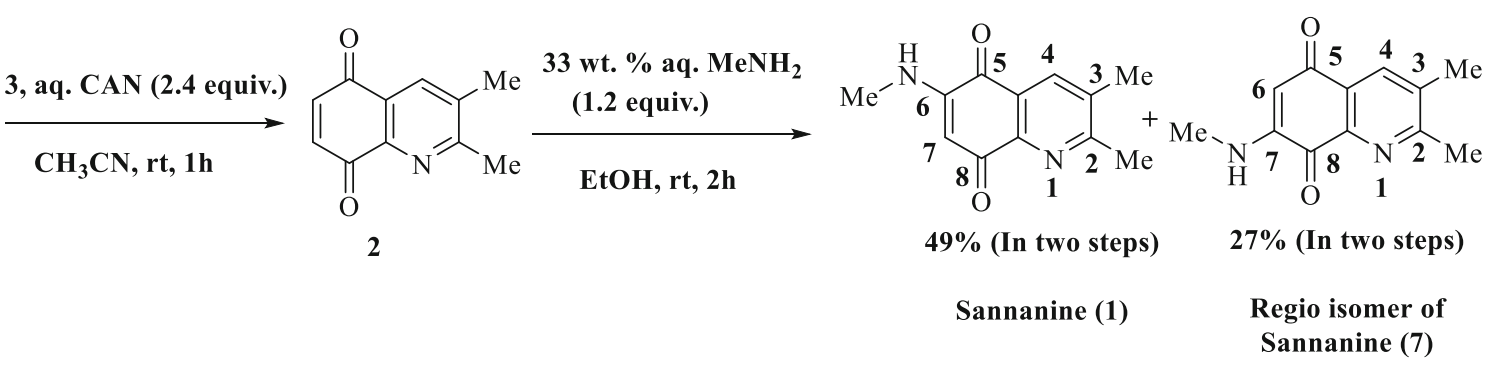
(1.2 equiv.)

EtOH, rt, 2h<smiles>COc1ccc(OC)c2nc(C)c(C)cc12</smiles>

$3,62 \%$<smiles>CCc1ccc2c(OC)ccc(OC)c2n1</smiles>

$6,34 \%$

Scheme 2. Synthesis of sannanine $\mathbf{1}$ and its regio isomer $\mathbf{7}$.

Table 1. ${ }^{1} \mathrm{H}\left(500 \mathrm{MHz}, \mathrm{CDCl}_{3}\right)$ and ${ }^{13} \mathrm{C} \mathrm{NMR}\left(125 \mathrm{MHz}, \mathrm{CDCl}_{3}\right)$ data of reported and synthesized compound 1.

\begin{tabular}{lcccc}
\hline Sl. No & $\delta_{H}$ (Reported) ppm & $\delta_{H}$ (Synthesized) & $\delta_{C}$ (Reported) ppm & $\delta_{C}$ (Synthesized) \\
\hline $\mathrm{NH}$ & $5.91(1 \mathrm{H}, \mathrm{brs})$ & $5.98(1 \mathrm{H}, \mathrm{brs})$ & - & - \\
2 & - & - & 164.4 & 164.4 \\
3 & - & - & 135.3 & 135.3 \\
4 & $8.01(1 \mathrm{H}, \mathrm{s})$ & - & 134.2 & 134.3 \\
$4 \mathrm{a}$ & - & - & 125.3 & 125.4 \\
5 & - & - & 181.78 & 181.80 \\
6 & - & $5.80(1 \mathrm{H}, \mathrm{s})$ & 148.3 & 148.4 \\
7 & $5.83(1 \mathrm{H}, \mathrm{s})$ & - & 101.1 & 101.1 \\
8 & - & - & 181.75 & 181.81 \\
$8 \mathrm{a}$ & - & $2.66(3 \mathrm{H}, \mathrm{s})$ & 146.7 & 146.8 \\
9 & $2.69(3 \mathrm{H}, \mathrm{s})$ & $2.39(3 \mathrm{H}, \mathrm{s})$ & 23.8 & 23.4 \\
10 & $2.40(3 \mathrm{H}, \mathrm{s})$ & & 19.3 & 19.3 \\
11 & $2.93(\mathrm{~d}, J=5.4 \mathrm{~Hz}, 3 \mathrm{H})$ & $2.92(\mathrm{~d}, J=5.6 \mathrm{~Hz}, 3 \mathrm{H})$ & 29.1 & 29.1 \\
\hline
\end{tabular}


<smiles>[R]NC1=CC(=O)c2cc(C)c(C)nc2C1=O</smiles><smiles>[R]#[W]Cc1ccccc1</smiles><smiles>Cc1cc2c(nc1C)C(=O)C=C(NCc1ccccc1)C2=O</smiles><smiles>[131In]</smiles><smiles>Cc1cc2c(nc1C)C(=O)C(NCc1ccccc1)=CC2=O</smiles>

$24 \%(9)$<smiles>[R]CCc1ccccc1</smiles><smiles>Cc1cc2c(nc1C)C(=O)C=C(NCCc1ccccc1)C2=O</smiles><smiles>Cc1cc2c(nc1C)C(=O)C(NCCc1ccccc1)=CC2=O</smiles>

$66 \%(10)$

$30 \%$ (11)<smiles>[R]CCc1ccc(OC)cc1</smiles><smiles>[R]=C=CCc1c[nH]c2ccccc12</smiles><smiles>Cc1cc2c(nc1C)C(=O)C=C(NCCc1c[nH]c3ccccc13)C2=O</smiles>

$48 \%$ (14)<smiles>C=CCNC1=CC(=O)c2nc(C)c(C)cc2C1=O</smiles>

$59 \%(16)$<smiles>C#CCNC1=CC(=O)c2nc(C)c(C)cc2C1=O</smiles>

$52 \%(18)$<smiles>Cc1cc2c(nc1C)C(=O)C(NCCc1c[nH]c3ccccc13)=CC2=O</smiles>

$27 \%$ (15)<smiles>C=CCNC1=CC(=O)c2cc(C)c(C)nc2C1=O</smiles>

$8 \%(17)$<smiles>C#CCNC1=CC(=O)c2cc(C)c(C)nc2C1=O</smiles>

$31 \%(19)$

Scheme 3. Synthesis of analogues 8-19 of sannanine 1. 
While considering the biological potent of indole containing compounds, tryptamine core was used in the analogues of $\mathbf{1 4}$ and $\mathbf{1 5}$. Gratifyingly, all other analogues have reached in moderate to good yields. The major intention of choosing the substitution in the quinoline5,8-dione core was to develop the method to construct many natural products as well as core structure of the biologically potent molecules using the quinoline-5,8dione as the main core.

\section{Conclusions}

In summary, the synthesis of sannanine and its analogues have been accomplished for the first time. The overall yield of the sannanine of $30 \%$ was achieved using easily affordable starting materials in a concise manner.

\section{Supplementary Information (SI)}

For newly synthesized compounds, ${ }^{1} \mathrm{H} /{ }^{13} \mathrm{C} /{ }^{13} \mathrm{C}$ DEPT NMR spectral data are depicted in Supplementary Information. Supplementary Information is available at www.ias.ac.in/ chemsci.

\section{Acknowledgements}

BN thanks to the UGC-SRF for fellowship. Both RN and BN acknowledge DST-SERB project number EMR/2016/ 007015/OC for the funding.

\section{References}

1. (a) Porter $\mathrm{T}$ H, Bowman C M and Folkers K 1973 antimetabolites of Coenzyme Q. 16. New Alkylmercaptoquinones Having Antimalarial Curative Activity J. Med. Chem. 16 115; (b) Rao K V, Biemann K and Woodward R B 1963 Studies of the Cycloheptatriene-Norcaradiene Isomerism in Some Novel Steroids J. Am. Chem. Soc. 85 2532; (c) Doyle T W, Balitz D M, Grulich R E, Nettleton D E, Gould S J, Tann C-H and Moews A E 1981 Structure Determination of Lavendamycin - a new Antitumor Antibiotic from Streptomyces Lavendulae Tetrahedron Lett. 22 4595; (d) Boger D L, Yasuda M, Mitscher L A, Drake S D, Kitos S D, Kitos P A and Thompson S C 1987 Streptonigrin and Lavendamycin Partial Structures. Probes for the Minimum, Potent Pharmacophore of Streptonigrin, Lavendamycin, and Synthetic Quinoline5,8-diones J. Med. Chem. 301918
2. (a) Fryatt T, Pettersson H I, Gardipee W T, Bray K C, Green S J, Salwin A M Z, Beall H D and Moody C J 2004 Novel Quinolinequinone Antitumor Agents: Structure-metabolism Studies with NAD(P)H: Quinone Xidoreductase (NQO1) Bioorg. Med. Chem. 12 1667; (b) Hargreaves R, David C L, Whitesell L and Skibo E B 2003 Design of Quinolinedione-Based Geldanamycin Analogues Bioorg. Med. Chem. Lett. 13 3075; (c) Argreaves R H J, David C L, Whitesell L J, LaBarbera D V, Jamil A, Chapuis J C and Skibo E B 2008 Discovery of Quinolinediones Exhibiting a Heat Shock Response and Angiogenesis Inhibition J. Med. Chem. 51 2492; (d) Behforouz M and Merriman R L 1995 Quinoline-5,8diones and Methods of Using them USOO5712289A 1; (e) Pearce A N, Chia E W, Berridge M V, Clark G R, Harper J L, Larsen L, Maas E W, Page M J, Perry N B, Webb V L and Copp B R 2007 Anti-inflammatory Thiazine alkaloids Isolated from the New Zeeland Ascidian Aplidium sp.: Inhibitors of the Neutrophil Respiratory Burst in a Model of Gouty Arthritis J. Nat. Prod. 70 936

3. (a) Machura B, Switlicka A, Wolff M, Tanak D, Musio R, Polanski J and Kruszynki R 2011 Novel Tricarbonyl Rhenium Complexes of 5,8-Quinolinedione Derivatives Synthesis, Spectroscopic Characterisation, X-ray Structure and DFT Calculations J. Organomet. Chem. 696 731; (b) Yoshida K, Noma Y and Ueno Y 1994 Synthesis and Properties of 6-Substituted Quinoline-5,8Dione Colour Formers J. Chem. Soc. Perkin T. 1 2521

4. (a) Seradj H, Cai W, Erasga N O, Chenault D V, Knuckles K A, Ragains J R and Behforouz M 2004, Total Synthesis of Novel 6-Substituted Lavendamycin Antitumer Agents Org. Lett. 6 473; (b) Blanco M D M, Avendano C and Menendez J C 1999 Concise synthesis of tetrahydro derivatives of the pyrido[2,3-b]acridine and pyrido[3,2-b]acridine ring systems Tetrahedron $\mathbf{5 5}$ 12637

5. Li Y, Zheng D, Li Z, Han L, Cui X, Lang L, Li M, Wang Z, Zhao J and Huang X 2009 Sannanine, A New Cytotoxic Alkaloid From Streptomyces Sannanensis J. Antibiot. 62 647

6. (a) Fernandez M, Lopez F, Tapia R and Valderrama J A 1989 Studies on the Synthesis of Fused Heterocycles From Ortho-Amino carbonyl Compounds Synth. Commun. 19 3087; (b) Blanco M D M, Avendano C, Cabezas N and Menendez J C 1993 A Friedlander Approach to 3Substituted 2,5,8-(1 H)-Quinolinetrione Heterocycles 36 1387

7. Hargreaves R H J, David C L, Whitesell L J, LaBarbera D V, Jamil A, Chapuis J C and Skibo E B 2008 Discovery of Quinolinediones Exhibiting a Heat Shock Response and Angiogenesis Inhibition J. Med. Chem. 512492 